\title{
XLVII. On the applicability of Lagrange's equations in certain cases of fluid-motion
}

\author{
John Purser M.A.
}

To cite this article: John Purser M.A. (1878) XLVII. On the applicability of Lagrange's equations in certain cases of fluid-motion, Philosophical Magazine Series 5, 6:38, 354-359, DOI: $10.1080 / 14786447808639525$

To link to this article: http://dx.doi.org/10.1080/14786447808639525

曲 Published online: 13 May 2009.

Submit your article to this journal $\lceil\pi$

Џ Article views: 2

Q View related articles $\square$ 
XLVII. On the Applicalility of Lagrange's Equations in certain Cases of Fluid-Motion. By JoHn Purser, M.A., Professor of Mathematics in the Queen's Colleye, Belfast*.

THE ordinary condition for the applicability of Lagrange's 1 equations to the motion of a system is that the position of all its parts be determined as a function of the generalized coordinates or parameters which enter into these equations. When such is not the case, even though the kinetic energy may be expressible in terms of these coordinates and their differential coefficients with respect to the time, the Lagrangian equations of motion are known not to be in general valid.

A familiar illustration of this is afforded by the motion of a rigid body rolling on a plane so rough as to prevent sliding. Here it is evident that the kinetic energy can be expressed in terms of three coordinates defining the angular position of the body and their differential coefficients. It is not, however, possible to express the position of all the points of the body in terms of these coordinates only; and accordingly the use of Lagrange's equations in terms of these coordinates is known to lead to erroneous results. It becomes, therefore, a matter of considerable interest to inquire into the grounds which justify recent important applications of these equations to sundry problems of hydrokinetics, relating to the motion of rigid bodies in an incompressible frictionless fluid.

This case, in fact, is in so far similar to that of the rolling body already alluded to, that while the kinetic energy of the system can be expressed (in virtue of Green's theorem) in terms of the limited number of parameters which define the position of the rigid bodies, it is clear from the smallest consideration that these parameters do not determine the position of the particles of the fluid. It would certainly seem, then, that we are not entitled prima facie to assume the validity of Lagrange's equations when applied to such problems, and that, if their use be here justifiable, it must be in virtue of special reasons. To endeavour to supply the proof which is thus seen to be requisite is the object of the present communication.

Given a number of rigid bodies moving in a frictionless incompressible fluid, whether infinitely extended or enclosed in a rigid envelope; given also that the motion at one epoch is irrotational, and therefore always so,--then by D'Alembert's principle,

$$
\Sigma d m\{\ddot{x} \delta x+\ddot{y} \delta y+\ddot{z} \delta z\}+\delta \mathrm{V}=0, \quad .
$$

* Communicated by the Author, having been read before the British Association at Dublin, August 1878. 
where $\mathrm{V}$ denotes the potential energy due to the applied forces which act on the bodies. $\delta x, \delta y, \delta z$, as far as they apply to the particles of the bodies, can, of course, be expressed in terms of $\delta q_{1}, \delta q_{2}, \& c$., the variations of the generalized coordinates determining the position of the bodies. And the same holds true for the particles of the fluid, provided we suppose the displacements irrotational. The above equation may therefore be written

$$
\mathrm{Q}_{1} \delta q_{1}+\dot{\mathrm{Q}}_{2} \delta q_{2} \ldots+\frac{d \mathrm{~V}}{d q_{1}} \delta q_{1}+\frac{d \mathrm{~V}}{d q_{2}} \delta q_{2} \ldots=0
$$

and the equations of motion are

$$
\mathrm{Q}_{1}+\frac{d \mathrm{~V}}{d g_{1}}=0, \quad \mathrm{Q}_{2}+\frac{d \mathrm{~V}}{d q_{2}}=0, \& \mathrm{c} .
$$

To obtain $Q_{1}$, suppose

then

$$
\delta q_{2}=0, \quad \delta q_{3}=0, \& \mathrm{c} .
$$

Now if

$$
\begin{aligned}
& \Sigma d m(\ddot{x} \delta x+\ddot{y} \delta y+\ddot{z} \delta z) \\
= & \Sigma d m \frac{d}{d t}(\dot{x} \delta x+\dot{y} \delta y+\dot{z} \delta x) \\
- & \Sigma d m\left(\dot{x} \frac{d}{d t} \delta x+\dot{y} \frac{d}{d t} \delta y+\dot{z} \frac{d}{d t} \delta z\right) .
\end{aligned}
$$

$$
\delta x=a_{1} \delta q_{1}, \quad \delta y=b_{1} \delta q_{1}, \quad \delta z=c_{1} \delta q_{1}, \& c_{0},
$$

when $a_{1}, b_{1}, c_{1}$ are functions of the $q$ 's and the coordinates $x, y, z$, then

$$
\begin{gathered}
\dot{x}=a_{1} \dot{q}_{1}, \quad \dot{y}=b_{1} \dot{q}_{1}, \quad \dot{z}=c_{1} \dot{q}_{1}, \& c . ; \\
\therefore a_{1}=\frac{d \dot{x}}{d \dot{q}_{1}}, \quad b_{1}=\frac{d \dot{y}}{d \dot{q}_{1}}, \quad \& c . ; \\
\therefore \Sigma d m \frac{d}{d t}(\dot{x} \delta x+\dot{y} \delta y+\dot{z} \delta z) \\
=\delta q_{1} \Sigma d m \frac{d}{d t}\left(\dot{x} \frac{d \dot{x}}{d \dot{q}_{1}}+\dot{y} \frac{d \dot{y}}{d \dot{q}_{1}}+\dot{z} \frac{d \dot{z}}{d \dot{q}_{1}}\right)=\delta q_{1} \frac{d}{d t}\left(\frac{d \mathrm{~T}}{d \dot{q}_{1}}\right) .
\end{gathered}
$$

If now we are justified in assuming that

$$
\begin{aligned}
& \Sigma d m\left(\dot{x} \frac{d}{d t} \delta x+\dot{y} \frac{d}{d t} \delta y+\dot{z} \frac{d}{d t} \delta z\right) \\
= & \Sigma d m\left(\dot{x} \delta \dot{x}+\begin{array}{c}
\left.\delta y+\dot{z} \delta^{z}\right), \\
2 \mathrm{~A} 2
\end{array}\right.
\end{aligned}
$$


then, since this latter expression $=\delta q_{1} \frac{d \mathrm{~T}}{d q_{1}}$, we get at once Lagrange's equation,

$$
\frac{d}{d t}\left(\frac{d \mathrm{~T}}{d \dot{q}_{1}}\right)-\frac{d \mathrm{~T}}{d q_{1}}+\frac{d \mathrm{~V}}{d q_{1}}=0 .
$$

It remains only to consider why the above assumption is legitimate. As far as the integral applies to the particles of the rigid bodies, since $\frac{d}{d t} \delta x=\delta \frac{d x}{d t}$ for each particle, the transformation is obviously justified. For the fluid, on the other hand, it is evident that, for an individual particle, $\frac{d}{d t} \delta x$ is not $=\delta \frac{d x}{d t}$. (This may be seen at once by considering the case of the motion of a flat piece of cardboard in a fluid, and supposing the time displacement perpendicular to its plane and the arbitrary displacement in its plane.)

To examine the meaning of the differences $\left(\frac{d}{d t} \delta x-\delta \frac{d x}{d t}\right) d t$, \&c., let us suppose that the generalized coordinates are so taken that one only of the coordinates, say $q_{2}$, alters with the time, so that the actual time displacement may be treated in the same way as a possible displacement $\delta q_{2}$.

The above may accordingly be written $\delta_{2} \delta_{1} x-\delta_{1} \delta_{2} x$, where $\delta_{1}, \delta_{2}$ correspond to the variations $\delta q_{1}, \delta q_{2}$.

Consider any point of the fluid $A$. Suppose the dispacement $\delta q_{1}$ followed by the displacement $\delta q_{2}$, and let the answering positions of $A$ be $\mathrm{B}$ and C. Again, suppose the displacement $\delta q_{2}$ followed by $\delta q_{1}$, and let the answering positions of $A$ be $D$ and $E$. $E$ will not coincide with $\mathrm{C}$, but the displacement is that $A$

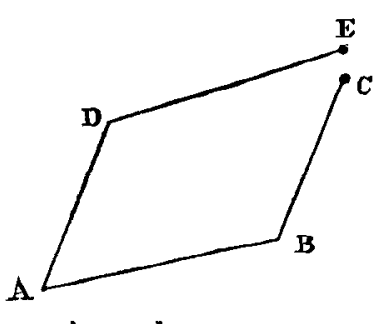
which has for its projections the expressions above.

For, projection of $\mathrm{EC}=$ projection of $\mathrm{DE}$ - projection of $\mathrm{AB}$ - (projection of $\mathrm{BC}$-projection of $\mathrm{AD})$

$$
=\delta_{2} \delta_{1} x-\delta_{1} \delta_{2} x \text {. }
$$

The bodies, however, after these two compound displacements are in identical positions; and consequently the displacements of the fluid $\left(\delta_{2} \delta_{1}-\delta_{1} \delta_{2}\right) x$, \&c., correspond to irrotational displacements of the fluid compatible with a position of the 
bodies momentarily fixed. We have then to show that, for such a displacement,

$$
\Sigma d m(\dot{x} \delta x+\dot{y} \delta y+\dot{z} \delta z)=0 .
$$

This is evident; for the work done by the momenta of the particles of the system must for any possible displacement be equal to that done by the impulse; and the latter in this case vanishes, as the bodies remain fixed.

The same thing may be shown analytically thus:-

$$
\begin{aligned}
& \Sigma d m(\dot{x} \delta x+\dot{y} \delta y+\dot{z} d z) \\
= & \Sigma d m\left(\frac{d \phi}{d x} \delta x+\frac{d \phi}{d y} \delta y+\frac{d \phi}{d z} \delta z\right) \\
= & \iint d \mathrm{~S} \cdot \rho \cdot \phi \cdot \delta n \\
& -\iiint d x d y d z \cdot \rho \cdot \phi \cdot\left(\frac{d \delta x}{d x}+\frac{d \delta y}{d y}+\frac{d \delta z}{d z}\right) .
\end{aligned}
$$

The first term vanishes, since the motion of the fluid in contact with the bodies is tangential; the second, since the fluid is incompressible.

\section{Addendum.}

Sir William Thomson has shown, in his paper on VortexMotion (Trans. R. S. E. vol. xxv.), that if one or more solid bodies are moving in an infinitely extended frictionless incompressible fluid, the motion of the fluid being supposed at one instant and therefore always irrotational, the impulse (i. e. the system of forces which would at any instant, if applied to the solids, generate the motion of the system of solids and fluids) would, if applied to a rigid body, represent a constant motive. It may be interesting to show that this conclusion follows directly from the Lagrangian equations.

First, let there be only one rigid body. Take two systems of coordinate axes-the first $(\mathrm{OX}, \mathrm{OY}, \mathrm{OZ})$ fixed in space, the second $\left(0^{\prime} \mathbf{X}^{\prime}, O^{\prime} Y^{\prime}, O^{\prime} Z^{\prime}\right)$ attached to the body.

Let $u, v, w$ be the components of the velocity of $\mathrm{O}^{\prime}$ estimated along the moving axes ;

$p, q, r$ the rotations of the body round these axes;

$\phi, \psi, \theta$ the usual angles denoting the position of the moving axes with respect to the fixed axes;

$x, y, z$ the coordinates of $\mathrm{O}^{\prime}$ with respect to the fixed axes.

Then, taking as generalized coordinates $x, y, z, \phi, \Psi, \theta$, we 
358 Prof. J. Purser on the Applicability of Lagrange's

should have, were continuous forces applied to the body,

$$
\begin{aligned}
& \frac{d}{d t} \cdot \frac{d \mathrm{~T}}{d \dot{x}}-\frac{d \mathrm{~T}}{d x}=x \text {-component of forces, } \\
& \frac{d}{d t} \cdot \frac{d \mathrm{~T}}{d \dot{\psi}}-\frac{d \mathrm{~T}}{d \psi}=\text { moment of forces round } \mathrm{O}^{\prime} \mathrm{Z},
\end{aligned}
$$

$\mathrm{O}^{\prime} \mathrm{Z}$, being a parallel through $\mathrm{O}^{\prime}$ to $\mathrm{OZ}$.

It follows, integrating through the short interval of time during which the instantaneous impulse would act, that

$$
\begin{aligned}
& \frac{d \mathrm{~T}}{d \dot{x}}=\text { component of impulse along } \mathrm{OX}, \\
& \frac{d \mathrm{~T}}{d \dot{y}}= \\
& \text { " } \\
& \frac{d \mathrm{~T}}{d \dot{z}}=\quad, \quad " \quad, \quad \mathrm{OZ}, \\
& \frac{d \mathrm{~T}}{d \dot{\psi}}=\text { component of moment of impulse round } O^{\prime} Z_{,} \text {. }
\end{aligned}
$$

In the actual motion

$$
\frac{d}{d t} \cdot \frac{d \mathrm{~T}}{d \dot{x}}-\frac{d \mathrm{~T}}{d x}=0 ;
$$

$\therefore$, since $\mathrm{T}$ does not depend on $x, y, z$,

$$
x \text {-component of impulse }=\frac{d \mathrm{~T}}{d \dot{x}}=\text { constant, }
$$

and similarly for the $y$-component and the $z$-component.

Let us now proceed to find the physical meaning of $\frac{d T}{d \psi}$, and interpret the equation

$$
\frac{d}{d t} \cdot \frac{d \mathrm{~T}}{d \dot{\psi}}-\frac{d \mathrm{~T}}{d \psi}=0
$$

It is clear from Green's theorem that $\mathrm{T}$ can be expressed as a quadratic function of $p, q, r, u, v, w$ with constant coefficients ;

$$
\begin{aligned}
\therefore \frac{d \mathrm{~T}}{d \psi} & =\frac{d \mathrm{~T}}{d p} \cdot \frac{d p}{d \psi}+\frac{d \mathrm{~T}}{d q} \cdot \frac{d q}{d \psi}+\frac{d \mathrm{~T}}{d r} \cdot \frac{d r}{d \Psi} \\
& +\frac{d \mathrm{~T}}{d u} \cdot \frac{d u}{d \psi}+\frac{d \mathrm{~T}}{d v} \cdot \frac{d v}{d \psi}+\frac{d \mathrm{~T}}{d w} \cdot \frac{d w}{d \psi}
\end{aligned}
$$

When $p, q, r$ are expressed in terms of $\phi, \psi, \theta$, their coeffcients do not involve the precessional angle $\psi$. Therefore the first three terms vanish. 
Again,

$$
\frac{d \mathrm{~T}}{d u}, \frac{d \mathrm{~T}}{d v}, \frac{d \mathrm{~T}}{d w}
$$

are the force-components of the impulse with respect to $0^{\prime} X^{\prime}$, $\mathrm{O}^{\prime} \mathrm{Y}^{\prime}, \mathrm{O}^{\prime} \mathrm{Z}^{\prime}$;

$$
\frac{d u}{d \psi}, \frac{d v}{d \psi}, \frac{d w}{d \psi}
$$

correspond to the displacement of a vector $u, v, w$ fixed in space with respect to the moving axes, owing to the motion $d \psi$ of the latter;

$$
\begin{aligned}
& \therefore \frac{d \mathrm{~T}}{d \psi}=\mathrm{R} \cdot \frac{\text { component along } \mathrm{R} \text { of relative displacement of }}{d \psi} \\
&=- \text { moment of a force } \mathrm{R} \text { applied at end of vector } \\
& u, v, w \text { round axis } \mathrm{O}^{\prime} \mathrm{Z}_{i} ;
\end{aligned}
$$

$\therefore$ Lagrange's equation

means that

$$
\frac{d}{d t} \cdot \frac{d \mathrm{~T}}{d \dot{\psi}}-\frac{d \mathrm{~T}}{d \dot{\psi}}=0
$$

$\frac{d}{d t}$ (moment of impulse round $\mathrm{O}^{\prime} \mathrm{Z}_{\mathrm{l}}$ )

+ moment of force of impulse applied at end of vector $u, v, w$ round the same line $=0$.

The left-hand member is obviously

$$
\left.=\frac{d}{d t} \text { (moment of impulse round } \mathrm{OZ}\right) \text {; }
$$

$\therefore$ this last moment is constant, and the moments round $\mathrm{OX}$ and $\mathrm{OY}$ are also constant.

Secondly, let there be more rigid bodies than one. We can now assume as the generalized coordinates the same as we have just taken which have reference to one of the bodies, together with other coordinates depending entirely upon the relative position of the rigid bodies amongst themselves; then $\frac{d \mathrm{~T}}{d \dot{x}}$ and $\frac{d \mathrm{~T}}{d \dot{\psi}}$ are evidently the force-components along the axis $\mathrm{OX}$ and the moment round the axis $\mathrm{O}^{\prime} \mathrm{Z}$, of the whole impulse, and the reasoning runs as before. 